\title{
Travelling Waves Solution of the Unsteady Flow Problem of a Rarefied Nonhomogeneous Charged Gas Bounded by an Oscillating Plate
}

\author{
Taha Zakaraia Abdel Wahid \\ Mathematics and Statistics Department, El-Madina Higher Institution of Administration and Technology, El-Madina Academy, Egypt \\ Correspondence should be addressed to Taha Zakaraia Abdel Wahid; taha_zakaraia@yahoo.com
}

Received 21 May 2013; Revised 17 September 2013; Accepted 22 September 2013

Academic Editor: Tirivanhu Chinyoka

Copyright ( 2013 Taha Zakaraia Abdel Wahid. This is an open access article distributed under the Creative Commons Attribution License, which permits unrestricted use, distribution, and reproduction in any medium, provided the original work is properly cited.

\begin{abstract}
The extension of the previous paper (Abdel Wahid and Elagan, 2012) has been made for a nonhomogeneous charged rarefied gas mixture (two-component plasma) instead of a single electron gas. Therefore, the effect of the positive ion collisions with electrons and with each other is taken into consideration, which was ignored, as an approximation, in the earlier work. Thus, we will have four collision terms (electron-electron, electron-ion, ion-ion, and ion-electron) instead of one term, as was studied before. These collision terms are added together with a completely additional system of differential equations for ions. This study is based on the solution of the Bhatnager-Gross-Krook (BGK) model of the Boltzmann kinetic equation coupled with Maxwell's equations. The initial-boundary value problem of the Rayleigh flow problem applied to the system of the two-component plasma (positive ions + electrons), bounded by an oscillating plate, is solved. This situation, for the best of my knowledge, is presented from the molecular viewpoint for the first time. For this purpose, the traveling wave solution method is used to get the exact solution of the nonlinear partial differential equations. In addition, the accurate formula of the whole four-collision frequency terms is presented. The distinction and comparisons between the perturbed and the equilibrium velocity distribution functions are illustrated. Definitely, the equilibrium time for electrons and for ions is calculated. The relation between those times and the relaxation time is deduced for both species of the mixture. The ratios between the different contributions of the internal energy changes are predicted via the extended Gibbs equation for both diamagnetic and paramagnetic plasmas. The results are applied to a typical model of laboratory argon plasma.
\end{abstract}

\section{Introduction}

A development of the previous paper [1] is introduced. The nonstationary Krook kinetic equation model for a rarefied charged binary gas mixture is solved, instead of the single gas. Analytically, the Bhatnager-Gross-Krook (BGK) model of the kinetic equation is applied. The travelling wave solution method is used to get the exact solution of the nonlinear partial differential equations. These equations were produced from applying the moment method to the unsteady BGK equation. Now we should solve eight nonlinear partial differential equations, which represent an arduous task. This situation, for the best of our knowledge, is presented in this paper for the first time. The unsteady solution for a binary charged gas mixture (two-component plasma) gives the problem a great generality and more applications. Taking into consideration the effect of the positive ions on the behavior of electrons, which was not observed in the previous work [1], this effect becomes a pressing mater in some physical situation; see, for example, [2]. The problem is investigated, with the new circumstance, to follow the behavior of the macroscopic properties of the gas such as the mean velocity, the shear stress, and the viscosity coefficient. The important quantities together with both the induced electric and magnetic fields are investigated for the two-component mixture, with respect to both distance and time. This new study is done to examine the behavior of electrons and positive ions in the microscale. This is performed using the kinetic Boltzmann equation coupled with Maxwell's equation. An important novel thermodynamic treatment of the system is concluded. 
The behavior of the nonequilibrium thermodynamic distribution functions for both positive ions and electrons, in different cases, is illustrated. Such treatment holds for the first time for a nonhomogeneous charged gas mixture bounded by oscillating plate. The calculated velocities are substituted into the corresponding two-stream Maxwellian distribution functions permitting us to investigate the nonequilibrium thermodynamic properties of the system (gas particles + the oscillating plate). The entropy, entropy flux, entropy production, thermodynamic forces, and kinetic coefficients are obtained. The verification of the Boltzmann H-theorem, Le Chatelier principle, and the second law of thermodynamic is examined. The ratios between the different contributions of the internal energy change based upon the total derivatives of the extensive parameters estimated via the Gibbs formula.

The behavior of charged gases in nonequilibrium states has received considerable attention from the standpoint of understanding the characteristics of nonequilibrium phenomena [3]. The kinetic theory has contributed not only to the understanding of nonequilibrium transport phenomena in gases, but also to the development of general nonequilibrium statistical physics. It is well accepted that the Boltzmann equation [3-8] is one of the most reliable kinetic models for describing nonequilibrium phenomena in gas phase. Following its success and usefulness, The Boltzmann equation is widely used in order to describe various gas-phase transport phenomena such as plasma gases, granular gases, polyatomic gases, relativistic gases, thermal radiation affected by gases, and chemically reacting gases [9-15]. The kinetic equation of gas flow based on the Boltzmann equation has obvious peculiarities in comparison with the macroscopic description found by using the Navier-Stokes equations; see [4, 13].

For planar flows, the oscillating Couette problem has many analogies with Stokes' second problem, which involves a flat plate oscillating in an unbounded medium. Stokes [16] first solved this problem in 1851 and later it was solved by Rayleigh [17] in 1911. Stokes' second problem is one of the simplest configurations to study the behavior of a nonequilibrium gas responding to a plate oscillating in its own plane [18]. A comprehensive study has been carried out with the linearized Bhatnagar-Gross-Krook (BGK) equation over a wide range of Knudsen numbers [19]. The effect of the oscillation frequency on the amplitudes and phases of the velocity and shear stress was reported. In order to improve our understanding of time-periodic, shear driven gas flows, oscillatory Couette flow provides an ideal test case. It has been studied extensively using kinetic theory (Sharipov and Kalempa [20]; Park et al. [21]; Hadjiconstantinou [22]; Emerson et al. [23]; Doi [24]). A brief comparison of the linearized R13 (LR13) equations with DSMC data (Hadjiconstantinou [22]) was carried out by Taheri et al. [25]. Although oscillatory Couette flow is a simple case, it has many related applications in a variety of MEMS devices, for example, the Tang resonator (Tang et al. [26]). A proper understanding of flow phenomena in resonators will therefore help to improve the performance and quality factor (Frangi et al. [27]). In this study, the extended continuum governing equations are employed to compute the details of Stokes' second problem and oscillatory planar Couette flow in the early transition regime. The purpose of the paper is to analyze and assess the dynamic response capability of the higher-order moment equations for oscillatory flow and compare against the available kinetic data. Gu and Emerson [18] presented results using three different continuum-based models to study oscillatory flow in the transition regime. Data obtained from numerical solutions of the Boltzmann equation and the direct simulation Monte Carlo method are used to assess the ability of the continuum models to capture important rarefaction effects.

The objective of this paper is to seek the unsteady exact solution of the Boltzmann kinetic equation of an inhomogeneous charged gas mixture bounded by an oscillating plate, for the first time. The initial-boundary value problem of the Rayleigh flow problem, applied to the system of the twocomponent plasma (positive ions + electrons), is solved to determine the macroscopic parameters such as the mean velocity, shear stress, and viscosity coefficient, together with the induced electric and magnetic fields. The investigation of the collisions mutual effects of ions with electrons, in the form of the ion distribution function, is completely operated. The light will be shed upon the effects of collisions of electrons with ions in the form of the electrons distribution function. Using the estimated distribution functions, it is of fundamental physical importance to deliberate the irreversible thermodynamic behavior of the diamagnetic and paramagnetic plasma gas. The results are applied to a typical model of laboratory argon plasma. The agreements of the results with the preceding theoretical studies are clarified.

\section{The Physical Problem and Mathematical Formulation}

Let us assume that the upper half of the space $(y \geq 0)$, which is bounded by an infinite flat plate $(y=0)$, is filled with a nonhomogeneous mixture of charged particles of electrons and positive ions. An infinite flat plate is fixed at $y=0$, and parallel to the $x z$-plane. The plate oscillates harmonically in the $x$ direction with frequency $\beta$; that is, the velocity of the plate depends on the time $t$ as follows:

$$
V_{w}=R_{e}\left[U_{0} \exp (-i \beta t)\right]=U_{0} \operatorname{Cos}(\beta t),
$$

where the symbol $R_{e}$ denotes the real part of complex expression. The quantity $U_{0}$ is the velocity amplitude, which is assumed to be small when compared with the thermal molecular velocity $V_{T e}$ of the gas. The charged gas is initially in absolute equilibrium and the wall is at rest. Then the plate starts to oscillate suddenly in its own plane with a velocity $U_{0} \operatorname{Cos}(\beta t)$ along the $x$-axis ( $U_{0}$ and $\beta$ are constants). Moreover, the plate is considered impermeable, uncharged, and an insulator. The whole system (electrons + ions + plate) is kept at constant temperature. All physical quantities are defined in the Nomenclatures. 
Let the forces $\vec{f}_{e}$ and $\vec{f}_{i}$ acting on each electron and ion respectively be given by [28-30]

$$
\begin{gathered}
\vec{f}_{e}=-e \vec{E}_{e}-\frac{e}{c_{o}}\left(\vec{c} \wedge \vec{B}_{e}\right), \\
\vec{f}_{i}=e \vec{E}_{i}+\frac{e}{c_{o}}\left(\vec{c} \wedge \vec{B}_{i}\right) .
\end{gathered}
$$
follows:

The directions of the considered physical quantities are as

$$
\begin{array}{ll}
\vec{V}_{\alpha} \equiv\left(V_{x \alpha}, 0,0\right), & \vec{J}_{\alpha} \equiv\left(q_{\alpha} n V_{x \alpha}, 0,0\right), \\
\vec{E}_{\alpha} \equiv\left(E_{x \alpha}, 0,0\right), & \vec{B}_{\alpha} \equiv\left(0,0, B_{z \alpha}\right),
\end{array}
$$

where $\alpha=e$ for electrons and $\alpha=i$ for ions.

The $V_{x \alpha}, E_{x \alpha}, B_{z \alpha}$, and $J_{x \alpha}$ are functions of $y$ and $t$ that satisfie Maxwell's equations. The distribution function $F(y, c, t)$ of the particles for the plasma gas can be obtained from the Bhatnager-Gross-Krook (BGK) model [31] of the kinetic Boltzmann's equation as

$$
\begin{aligned}
\frac{\partial F_{e}}{\partial t} & +\vec{c} \cdot \frac{\partial F_{e}}{\partial \vec{r}}+\frac{\vec{f}_{e}}{m_{e}} \cdot \frac{\partial F_{e}}{\partial \vec{c}} \\
& =v_{e e}\left(F_{0 e}-F_{e}\right)+v_{e i}\left(\overline{F_{0 e}}-F_{e}\right) \text { for electrons, } \\
\frac{\partial F_{i}}{\partial t} & +\vec{c} \cdot \frac{\partial F_{i}}{\partial \vec{r}}+\frac{\vec{f}_{i}}{m_{i}} \cdot \frac{\partial F_{i}}{\partial \vec{c}} \\
& =v_{i i}\left(F_{0 i}-F_{i}\right)+v_{i e}\left(\overline{F_{0 i}}-F_{i}\right) \text { for ions, }
\end{aligned}
$$

where $F_{0 \alpha}=n_{\alpha}\left(2 \pi R T_{\alpha}\right)^{-3 / 2} \exp \left(-\left(\vec{c}-\vec{V}_{\alpha}\right)^{2} / 2 R T_{\alpha}\right)$ and $\overline{F_{0 \alpha}}=$ $n_{\alpha}\left(2 \pi R \overline{T_{\alpha}}\right)^{-3 / 2} \exp \left(-\left(\vec{c}-\overline{\vec{V}}_{\alpha}\right)^{2} / 2 R \overline{T_{\alpha}}\right)$.

The quantities $n_{\alpha}, \vec{V}_{\alpha}$ and $T_{\alpha}$ are the number density, mean drift velocity, and effective temperature obtained by taking moments of $F_{\alpha}$. Some latitude in the definition of $\overline{T_{\alpha}}$ and $\overline{\vec{V}_{\alpha}}$ is possible; one choice is in [32]

$$
\overline{T_{e}}=T_{i}, \quad \overline{T_{i}}=T_{e}, \quad \overline{\vec{V}_{e}}=\vec{V}_{i}, \quad \overline{\vec{V}_{i}}=\vec{V}_{e} .
$$

The particles are reflected from the plate with a full velocity accommodation; that is, the plasma particles are reflected with the plate velocity so that the boundary conditions are $V_{x 2 \alpha}(0, t)=U_{0} \operatorname{Cos}(\beta t)$ for $t>0$, where $V_{x 2 \alpha}=V_{x \alpha}$ as $c_{y}>$ 0 and $V_{x \alpha}$ is finite as $y \rightarrow \infty$ for both ions and electrons.

Substituting from (2), (3), and (6) into (4) and (5) one obtains

$$
\begin{gathered}
\frac{\partial F_{e}}{\partial t}+c_{y} \frac{\partial F_{e}}{\partial y}-\frac{e B_{e z}}{m_{e} c_{0}}\left(c_{y} \frac{\partial F_{e}}{\partial c_{x}}-c_{x} \frac{\partial F_{e}}{\partial c_{y}}\right)+\frac{e E_{e x}}{m_{e}} \frac{\partial F_{e}}{\partial c_{x}} \\
=v_{e e}\left(F_{0 e}-F_{e}\right)+v_{e i}\left(F_{0 i}-F_{e}\right), \text { for electrons, } \\
\frac{\partial F_{i}}{\partial t}+c_{y} \frac{\partial F_{i}}{\partial y}+\frac{e B_{i z}}{m_{i} c_{0}}\left(c_{y} \frac{\partial F_{i}}{\partial c_{x}}-c_{x} \frac{\partial F_{i}}{\partial c_{y}}\right)-\frac{e E_{x}}{m_{i}} \frac{\partial F_{i}}{\partial c_{x}} \\
=v_{i i}\left(F_{0 i}-F_{i}\right)+v_{i e}\left(F_{0 e}-F_{i}\right), \text { for ions, }
\end{gathered}
$$

where $v_{e e}, v_{e i}, v_{i i}$, and $v_{i e}$ are electron-electron, electron-ion, ion-ion, and ion-electron collision frequencies, respectively, which are given by [32-35]

$$
\begin{gathered}
\nu_{e e}=\left(\frac{4 \sqrt{\pi} n_{e} e^{4} \log \left[\Lambda_{e e}\right]}{3 \sqrt{m_{e}} K_{B}^{3 / 2} T_{e}^{3 / 2}}\right), \\
v_{e i}=\left(\frac{4 \sqrt{2 \pi} n_{i} e^{4} Z^{2} \log \left[\Lambda_{e i}\right]}{3 \sqrt{m_{e}} K_{B}^{3 / 2} T_{e}^{3 / 2}}\right), \\
v_{i i}=\left(\frac{4 \sqrt{\pi} n_{i} e^{4} Z^{4} \log \left[\Lambda_{i i}\right]}{3 \sqrt{m_{i}} K_{B}^{3 / 2} T_{i}^{3 / 2}}\right), \\
v_{i e}=\left(\frac{m_{e}}{m_{i}} \frac{4 \sqrt{2 \pi} n_{e} e^{4} Z^{2} \log \left[\Lambda_{i e}\right]}{3 \sqrt{m_{e}} K_{B}^{3 / 2} T_{e}^{3 / 2}}\right),
\end{gathered}
$$

where $\lambda_{D e}=\lambda_{D i}=\lambda_{D}, \log [\Lambda]=\log \left[4 \pi n \lambda_{D}^{3}{ }_{D}\right]$ and Zare the Coulomb Logarithms and the degree of ionization, respectively.

The model of the cone of influence suggested by Lee's moment method [36-40] for the solution of the Boltzmann's equation is employed here. Let us write the solution of (4) and (5), as suggested by Kashmarov in the form

$$
\begin{aligned}
& F_{\alpha} \\
& =\left\{\begin{array}{rr}
F_{1 \alpha}=n_{\alpha}\left(2 \pi R T_{\alpha}\right)^{-3 / 2}\left(1+\frac{c_{x} V_{x 1 \alpha}}{R T_{\alpha}}\right) & \exp \left(\frac{-c^{2}}{2 R T_{\alpha}}\right) \\
F_{2 \alpha}=n_{\alpha}\left(2 \pi R T_{\alpha}\right)^{-3 / 2}\left(1+\frac{c_{x} V_{x 2 \alpha}}{R T_{\alpha}}\right) & \exp \left(\frac{-c_{y}<0}{2 R T_{\alpha}}\right) \\
\text { for } c_{y}>0,
\end{array}\right.
\end{aligned}
$$

where $V_{x 1 \alpha}$ and $V_{x 2 \alpha}$ are four unknown functions of time $t$ and the distance variable $y$.

Using Grad's moment method multiplying (7) and (8) by $Q_{j}(\vec{c})$. Integrating over all values of $\vec{c}$, we can obtain the transfer equations for electrons and ions, respectively, in the form

$$
\begin{gathered}
\frac{\partial}{\partial t} \int Q_{j} F_{e} d \underline{c}+\frac{\partial}{\partial y} \int c_{y} Q_{j} F_{e} d \underline{c}+\frac{e E_{x e}}{m_{e}} \\
\times \int F_{e} \frac{\partial Q_{j}}{\partial c_{x}} d \underline{c}+-\frac{e B_{z e}}{m_{e} c_{0}} \int\left(c_{x} \frac{\partial Q_{j}}{\partial c_{y}}-c_{y} \frac{\partial Q_{j}}{\partial c_{x}}\right) d \underline{c} \\
=v_{e e} \int\left(F_{0 e}-F_{e}\right) Q_{j} d \underline{c}+v_{e i} \int\left(F_{0 i}-F_{e}\right) Q_{j} d \underline{c} \\
\frac{\partial}{\partial t} \int Q_{j} F_{i} d \underline{c}+\frac{\partial}{\partial y} \int c_{y} Q_{j} F_{i} d \underline{c}-\frac{e E_{i x}}{m_{i}} \\
\times \int F_{i} \frac{\partial Q_{j}}{\partial c_{x}} d \underline{c}+\frac{e B_{i z}}{m_{i} c_{0}} \int\left(c_{x} \frac{\partial Q_{j}}{\partial c_{y}}-c_{y} \frac{\partial Q_{j}}{\partial c_{x}}\right) d \underline{c} \\
=v_{i i} \int\left(F_{0 i}-F_{i}\right) d \underline{c}+v_{i e} \int\left(F_{0 e}-F_{i}\right) Q_{j} d \underline{c} .
\end{gathered}
$$


The integrals over the velocity distance are evaluated from the relation

$$
\begin{aligned}
\int Q_{j}(\vec{c}) F_{\alpha} d \underline{c}= & \int_{-\infty}^{\infty} \int_{-\infty}^{0} \int_{-\infty}^{\infty} Q_{j} F_{1 \alpha} d \underline{c} \\
& +\int_{-\infty}^{\infty} \int_{0}^{\infty} \int_{-\infty}^{\infty} Q_{j} F_{2 \alpha} d \underline{c},
\end{aligned}
$$

where $Q_{j}=Q_{j}(\vec{c}), j=1,2$, and $d \underline{c}=d c_{x} d c_{y} d c_{z}$, where $c_{x}, c_{y}$ and $c_{z}$ are the particles velocities components along $x$-, $y$-, and $z$-axes, respectively. Moreover, $E_{\alpha}$ and $B_{\alpha}$ may be obtained from Maxwell's equation, for electrons as follows:

$$
\begin{gathered}
\frac{\partial E_{x e}}{\partial y}-\frac{1}{c_{0}} \frac{\partial B_{z e}}{\partial t}=0 \\
\frac{\partial B_{z e}}{\partial y}-\frac{1}{c_{0}} \frac{\partial E_{x e}}{\partial t}-\frac{4 \pi e n_{e}}{c_{0}} V_{x e}=0 .
\end{gathered}
$$

For ions we obtain

$$
\begin{gathered}
\frac{\partial E_{x i}}{\partial y}-\frac{1}{c_{0}} \frac{\partial B_{z i}}{\partial t}=0 \\
\frac{\partial B_{z i}}{\partial y}-\frac{1}{c_{0}} \frac{\partial E_{x i}}{\partial t}+\frac{4 \pi e n_{i}}{c_{0}} V_{x i}=0
\end{gathered}
$$

where $n_{\alpha}=\int F_{\alpha} d \underline{c}, n_{\alpha} V_{x \alpha}=\int c_{x} F_{\alpha} d \underline{c}$, with the initial and boundary conditions

$$
\begin{gathered}
E_{x \alpha}(y, 0)=B_{z \alpha}(y, 0)=0 \\
E_{x \alpha}(y, t), B_{z \alpha}(y, t) \quad \text { are finite as } y \longrightarrow \infty .
\end{gathered}
$$

We introduce the dimensionless variables defined by

$$
\begin{aligned}
& t=t^{\prime} \tau_{i e}, \quad y=y^{\prime}\left(\frac{\tau_{i e} V_{T e}}{\sqrt{2 \pi}}\right), \\
& V_{x \alpha}=V_{x \alpha}^{\prime} V_{T e}, \quad \tau_{x y \alpha}=\tau_{x y \alpha}^{\prime} V_{T e}, \quad \mathrm{Ma}=\frac{U_{0}}{V_{T e}}, \\
& B_{z \alpha}=B_{z \alpha}^{\prime}\left(\frac{\sqrt{2 \pi} m_{e} c_{0}}{e \tau_{i e}}\right), \quad E_{x \alpha}^{\prime}=E_{x \alpha}^{\prime}\left(\frac{m_{e} V_{T e}}{e \tau_{i e}}\right), \\
& \rho_{\alpha}=n_{\alpha} m_{\alpha}, \quad V_{T e}=\sqrt{\frac{2 K_{B} T_{e}}{m_{e}}}, \\
& \varepsilon=\frac{m_{e}}{m_{i}}, \quad d U_{\alpha}=d U_{\alpha}{ }^{\prime}\left(K_{B} T_{e}\right), \\
& F_{j \alpha}=F_{j \alpha}{ }^{\prime} n_{e}\left(2 \pi R T_{e}\right)^{-3 / 2}, \quad j=0,1,2 .
\end{aligned}
$$

For $\mathrm{Ma}^{2} \ll 1$ (low Mach number), we can assume that the density and the temperature variation at each point of the flow and at any time are negligible; that is, $n_{\alpha}=1+O\left(\mathrm{Ma}^{2}\right)$ and $T_{\alpha}=1+O\left(\mathrm{Ma}^{2}\right)$. Put

$$
\begin{aligned}
& V_{x \alpha}=\frac{1}{2}\left(V_{x 1 \alpha}+V_{x 2 \alpha}\right), \\
& \tau_{x y \alpha}=\frac{P_{x y \alpha}}{\rho_{\alpha} U_{0} \sqrt{R T_{e} / 2 \pi}}=\left(V_{x 2 \alpha}-V_{x 1 \alpha}\right),
\end{aligned}
$$

where $P_{x y \alpha}$ is the shear stress [11] that is defined by $P_{x y \alpha}=$ $m \int\left(c_{x}-V_{x}\right) c_{y} F_{\alpha} d \underline{c}$.

Using the dimensionless variable, if we neglect terms of order $O\left(\mathrm{Ma}^{2}\right)$, (11) for $Q_{1}=c_{x}$ and $Q_{2}=c_{x} c_{y}$ is:

$$
\begin{gathered}
\frac{\partial V_{x e}^{\prime}}{\partial t^{\prime}}+\frac{\partial \tau_{x y e}^{\prime}}{\partial y^{\prime}}-E_{x e}^{\prime}=0 \\
\frac{\partial \tau_{x y e}^{\prime}}{\partial t^{\prime}}+2 \pi \frac{\partial V_{x e}^{\prime}}{\partial y^{\prime}}+\left(\frac{v_{e e}}{v_{i e}}+\frac{v_{e i}}{v_{i e}}\right) \tau_{x y e}^{\prime}=0 .
\end{gathered}
$$

Similarly, (12) becames:

$$
\begin{gathered}
\frac{\partial V_{x i}^{\prime}}{\partial t^{\prime}}+\frac{\partial \tau_{x y i}^{\prime}}{\partial y^{\prime}}+\varepsilon E_{x i}^{\prime}=0, \\
\frac{\partial \tau_{x y i}^{\prime}}{\partial t^{\prime}}+2 \pi \frac{\partial V_{x i}^{\prime}}{\partial t^{\prime}}+\left(\frac{v_{i i}}{v_{i e}}+\frac{v_{i e}}{v_{i e}}\right) \tau_{x y i}^{\prime}=0,
\end{gathered}
$$

with the initial and boundary conditions

$$
\begin{gathered}
V_{x \alpha}^{\prime}\left(y^{\prime}, 0\right)=\tau_{x y \alpha}^{\prime}\left(y^{\prime}, 0\right)=0, \\
2 V_{x \alpha}^{\prime}\left(0, t^{\prime}\right)+\tau_{x y \alpha}^{\prime}\left(0, t^{\prime}\right)=2 \mathrm{Ma} \operatorname{Cos}\left(\beta_{1} t^{\prime}\right) ; \\
V_{x}^{\prime}, \tau_{x y}^{\prime} \quad \text { are finite as } y \longrightarrow \infty, \beta_{1}=\beta \tau_{i e} .
\end{gathered}
$$

In the expressions for the transport coefficients mentioned previously, the fact that the plasma is neutral was used [33], writing $n=n_{e}=Z n_{i}, T=T_{e}=T_{i}$, and $Z=1$, which exploited the fact that the ratio $m_{e} / m_{i}$ is small. Thus, the ratio between the four distinguished collision frequencies can be rewritten in a form that referred to $v_{e e}$ in formulas (9)

$$
v_{e e}: v_{e i}: v_{i i}: v_{i e}=1: \sqrt{2}: \sqrt{\frac{m_{e}}{m_{i}}}: \frac{m_{e}}{m_{i}}=1: \sqrt{2}: \sqrt{\varepsilon}: \varepsilon .
$$

Therefore, $v_{e e} \sim v_{e i} \gg v_{i i} \gg v_{i e}$; the first of these gross inequalities arises because thermal ion speeds are less than thermal electron speeds by the factor $\sqrt{m_{e} / m_{i}}$ if $T_{e} \approx T_{i}$, and so the ions take longer period of time to meet each other. The second one reflects the fact that the electrons are not very effective in deflecting the much heavier ions.

For the sake of simplicity, henceforth, we drop the dash over the dimensionless variables. Therefore, we have the following initial-boundary value problem for electrons (neglecting the displacement current) [30]:

$$
\begin{gathered}
\frac{\partial V_{x e}}{\partial t}+\frac{\partial \tau_{x y e}}{\partial y}-E_{x e}=0 \\
\frac{\partial \tau_{x y e}}{\partial t}+2 \pi \frac{\partial V_{x e}}{\partial y}+\left(\frac{1}{\sqrt{\varepsilon}}+\sqrt{\frac{2}{\varepsilon}}\right) \tau_{x y e}=0 \\
\frac{\partial E_{x e}}{\partial y}-\frac{\partial B_{z e}}{\partial t}=0 \\
\frac{\partial B_{z e}}{\partial y}-\alpha_{0 e} V_{x e}=0, \quad \text { where } \alpha_{0 e}=\left(\frac{V_{T e}^{2} \tau_{i e}^{2} e^{2} n_{e}}{m_{e} c_{0}^{2}}\right),
\end{gathered}
$$


with the initial and boundary conditions

$$
\begin{gathered}
V_{x e}(y, 0)=\tau_{x y e}(y, 0)=E_{x e}(y, 0)=B_{z e}(y, 0)=0 \\
2 V_{x e}(0, t)+\tau_{x y e}(0, t)=2 \mathrm{Ma} \operatorname{Cos}\left(\beta_{1} t\right), \quad \text { for } t>0 \\
V_{x e}, \tau_{x y e}, E_{x e}, B_{z e} \quad \text { are finite as } y \longrightarrow \infty
\end{gathered}
$$

In addition, we have the following initial-boundary problem for ions (neglecting the displacement current):

$$
\begin{aligned}
& \frac{\partial V_{x i}}{\partial t}+\frac{\partial \tau_{x y i}}{\partial y}+\varepsilon E_{x i}=0 \\
& \frac{\partial \tau_{x y i}}{\partial t}+2 \pi \frac{\partial V_{x i}}{\partial y}+(\sqrt{\varepsilon}+1) \tau_{x y i}=0, \\
& \frac{\partial E_{x i}}{\partial y}-\frac{\partial B_{z i}}{\partial t}=0, \\
& \frac{\partial B_{z i}}{\partial y}+\alpha_{0 i} V_{x i}=0, \quad \text { where } \alpha_{0 i}=\left(\frac{V_{T e}^{2} \tau_{i e}^{2} e^{2} n_{i}}{m_{e} c_{0}^{2}}\right)
\end{aligned}
$$

Since $n_{i}=n_{e}$; thus, henceforth, we put $\alpha_{0 i}=\alpha_{0 e}=\alpha_{0}$.

We can reduce our basic (23)-(26), for electrons, after some analytical manipulations to a single equation as follows:

$$
\begin{gathered}
\frac{\partial^{4} V_{x e}(y, t)}{\partial t^{2} \partial y^{2}}-2 \pi \frac{\partial^{4} V_{x e}(y, t)}{\partial t^{4}}-A_{c} \frac{\partial^{3} V_{x e}(y, t)}{\partial t \partial y^{2}} \\
-\alpha_{0} \frac{\partial^{2} V_{x e}(y, t)}{\partial t^{2}}-\alpha_{0} A_{c} \frac{\partial V_{x e}(y, t)}{\partial t}=0,
\end{gathered}
$$

where $A_{c}=((1 / \sqrt{\varepsilon})+\sqrt{2 / \varepsilon})$. obtain

Similarly, the basic (28)-(31) can be reduced for ions to

$$
\begin{gathered}
\frac{\partial^{4} V_{x i}(y, t)}{\partial t^{2} \partial y^{2}}-2 \pi \frac{\partial^{4} V_{x i}(y, t)}{\partial t^{4}}-B_{c} \frac{\partial^{3} V_{x i}(y, t)}{\partial t \partial y^{2}} \\
-\alpha_{0} \varepsilon \frac{\partial^{2} V_{x i}(y, t)}{\partial t^{2}}-\alpha_{0} \varepsilon B_{c} \frac{\partial V_{x i}(y, t)}{\partial t}=0
\end{gathered}
$$

where $B_{c}=(\sqrt{\varepsilon}+1)$.

\section{Solution of the Initial-Boundary Value Problem}

The traveling wave solution method $[41,42]$ is used, considered

$$
\xi=l y-m t
$$

to make all the dependent variables as functions of $\xi$. Here $l$ and $m$ are transformation constants, which do not depend on the properties of the fluid but as parameters to be determined by the boundary and initial conditions [41]. Using (34) we obtain the derivatives

$$
\begin{array}{ll}
\frac{\partial}{\partial t}=-m \frac{\partial}{\partial \xi}, \quad \frac{\partial}{\partial y}=l \frac{\partial}{\partial \xi}, & \frac{\partial^{a}}{\partial t^{a}}=(-1)^{a} m^{a} \frac{\partial^{a}}{\partial \xi^{a}}, \\
\frac{\partial^{a}}{\partial y^{a}}=l^{a} \frac{\partial^{a}}{\partial \xi^{a}}, &
\end{array}
$$

where $a$ is a positive integer.

Substituting from (34)-(35) into (32), we have

$$
\begin{aligned}
& \left(m^{2} l^{2}-2 \pi m^{4}\right) \frac{d^{4} V_{x e}(\xi)}{d \xi^{4}}+A_{c} m l^{2} \frac{d^{3} V_{x e}(\xi)}{d \xi^{3}} \\
& -\alpha_{0} m^{2} \frac{d^{2} V_{x e}(\xi)}{d \xi^{2}}+\alpha_{0} A_{c} m \frac{d V_{x e}(\xi)}{d \xi}=0 .
\end{aligned}
$$

The boundary and initial conditions became

$$
\begin{gathered}
E_{x e}(\xi=0)=B_{z e}(\xi=0)=\tau_{x y e}(\xi=0)=0, \\
2 V_{x e}(\xi=-m)+\tau_{x y e}(\xi=-m)=2 \mathrm{MaCos}\left(\beta_{1}\right), \\
\text { at } y=0, \text { e.g., } t=1 ; \\
V_{x e}, \tau_{x y e}, E_{x e}, B_{z e} \quad \text { are finite as } \xi \longrightarrow-\infty .
\end{gathered}
$$

Now we have an ordinary differential Equation (36) with the boundary and initial conditions (37). Next, let us solve (33) by using the same tackling. Substituting from expressions (34)-(35) into (33), we get

$$
\begin{aligned}
& \left(m^{2} l^{2}-2 \pi m^{4}\right) \frac{d^{4} V_{x i}(\xi)}{d \xi^{4}}+B_{c} m l^{2} \frac{d^{3} V_{x i}(\xi)}{d \xi^{3}} \\
& \quad-\alpha_{0} \varepsilon m^{2} \frac{d^{2} V_{x i}(\xi)}{d \xi^{2}}+\alpha_{0} \varepsilon B_{c} m \frac{d V_{x i}(\xi)}{d \xi}=0,
\end{aligned}
$$

with the corresponding boundary and initial conditions

$$
\begin{gathered}
E_{x i}(\xi=0)=B_{z i}(\xi=0)=\tau_{x y i}(\xi=0)=0, \\
2 V_{x i}(\xi=-m)+\tau_{x y i}(\xi=-m)=2 \mathrm{Ma} \operatorname{Cos}\left(\beta_{1}\right) ; \\
V_{x i}, \tau_{x y i}, E_{x i}, B_{z i} \quad \text { are finite as } \xi \longrightarrow-\infty .
\end{gathered}
$$

The two ordinary, fourth order homogeneous differential equations (36) and (38) can be solved exactly by the help of symbolic computer software, with their boundary and initial conditions (37) and (39). The sought solutions will be applied to a typical model of laboratory argon plasma.

\section{The Investigation of the Behavior of the Internal Energy Change}

The studying of the behavior of the internal energy change for the physical systems presents a great importance in science. The extended Gibbs relation for electrons and ions is introduced to study the internal energy change for the system, 
based on the solution of the nonstationary Boltzmann equation [43]. It includes the electromagnetic field energy as a part of the complete energy balance. This procedure distinguishes the charged gas into paramagnetic and diamagnetic ones. If there are unpaired electrons in the molecular orbital diagram, the gas is paramagnetic. If all electrons are paired, the gas is considered as a diamagnetic one. We should write the internal energy balance, including the electromagnetic field energy, to get the work term in the first law of thermodynamics as follows.

(a) For paramagnetic plasma, the internal energy change is expressed in terms of the extensive quantities $S_{\alpha}, P_{\alpha}$, and $M_{\alpha}$, which are the thermodynamic coordinates corresponding to the conjugate intensive quantities $T_{\alpha}, E_{\alpha}$, and $B_{\alpha}$, respectively. The three contributions in the internal energy change in the Gibbs formula we have

$$
d U_{\alpha}=d U_{S \alpha}+d U_{\text {pol } \alpha}+d U_{\text {par } \alpha},
$$

where $d U_{S} \alpha=T d S_{\alpha}$ is the internal energy change due to the variation of the entropy, which is written in dimensionless form as

$$
S_{\alpha}=-\pi^{3 / 2}\left[\left(V_{x 1 \alpha}{ }^{2}+V_{x 2 \alpha}^{2}\right)-\frac{3}{2}\right],
$$

$d U_{\text {pol } \alpha}=E_{\alpha} d P_{\alpha}$ is the internal energy change due to variation of polarization, and $d U_{\text {para } \alpha}=B_{\alpha} d M_{\alpha}$ is the internal energy change due to the variation of magnetization, here $M$ is calculated from the equation [44]

$$
\frac{\partial S_{\alpha}}{\partial M_{\alpha}}=-\frac{B_{\alpha}}{T_{\alpha}} \Longrightarrow M_{\alpha}=-\int\left(\frac{T_{\alpha}}{B_{\alpha}} \frac{\partial S_{\alpha}}{\partial y}\right)_{t} d y .
$$

Introduce the dimensionless variables $U_{\alpha}^{\prime}=U_{\alpha} / K T_{\alpha}$, $M_{\alpha}^{\prime}=M_{\alpha}\left(1 / e_{\alpha} \tau_{e i} V_{T e}\right), p_{\alpha}^{\prime}=p_{\alpha}\left(1 / e_{\alpha} \tau_{e i} V_{T e}\right)$ in the Gibbs formula to get (after dropping the primes)

$$
d U_{\alpha}=d S_{\alpha}+f_{\alpha 1} E_{\alpha} d p_{\alpha}+f_{1 \alpha} B_{\alpha} d M_{\alpha} .
$$

(b) On the other hand, if the plasma is diamagnetic, the internal energy change due to the extensive variables $S, P$, and $B$ represents the thermodynamic coordinates conjugate to the intensive quantities $T$, $E$, and $M$, respectively; therefore, we have three contributions in the internal energy change in the Gibbs formula given by

$$
d U_{\alpha}=d U_{S \alpha}+d U_{\text {pol } \alpha}+d U_{\operatorname{dia} \alpha}
$$

where $d U_{\text {dia } \alpha}=-M_{\alpha} d B_{\alpha}$ is the internal energy change due to the variation of the induced magnetic induction, where $M_{\alpha}=T_{\alpha}\left(\partial S_{\alpha} / \partial B_{\alpha}\right)$. Hence, the dimensionless form for $d U$ in this case takes the following form:

$$
d U_{\alpha}=d S_{\alpha}+f_{\alpha 1} E_{\alpha} d p_{\alpha}-f_{\alpha 1} M_{\alpha} d B_{\alpha}
$$

where

$$
\begin{array}{r}
f_{1}=\left(\frac{m_{e} V_{T e}^{2}}{K T_{0}}\right), \quad d S_{\alpha}=\left(\frac{\partial S_{\alpha}}{\partial r}\right) \delta y+\left(\frac{\partial S_{\alpha}}{\partial t}\right) \delta t \\
\delta y=1, \delta t=4.35 \simeq t_{\text {eqe }}
\end{array}
$$

\section{Discussion}

In this problem, the unsteady behavior of an inhomogeneous mixture of charged gas, bounded by an oscillating plate, is investigated. This study is based on the kinetic theory via the BGK model of the Boltzmann equation. Our computations are performed according to typical data for argon plasma as a paramagnetic medium in the case of the argon gas losing single electrons subjected to the following conditions and parameters: $K_{B}=1.380710^{-16} \mathrm{erg} / \mathrm{K}, T_{0}=600 \mathrm{~K}, n_{e}=$ $710^{16} \mathrm{~cm}^{-3}, d=3.8410^{-8} \mathrm{~cm}$ (diameter of the argon atom); the electron rest mass, mass of ions, and charge are $m_{e}=$ $9.093 \times 10^{-28} \mathrm{gm}, m_{i}=6.633 \times 10^{-23} \mathrm{gm}, e=4.810^{-10} \mathrm{esu}$, which are used to calculate electron-ion relaxation time $\tau_{i e}=$ $1.4859 \times 10^{-10} \mathrm{sec}$. The dimensionless parameter is $\alpha_{0}=7.9 \times$ $10^{-2}$ and the mean free path of the electrons $\lambda_{i e}=2.110^{-3} \mathrm{~cm}$ compared to the electron Debye length.

Consider $\lambda_{D e}=\left(K_{B} T_{0} / 4 \pi n_{e} e^{2}\right)^{1 / 2}=6.39 \times 10^{-7} \mathrm{~cm}$, $f_{1}=2$. Using the idea of the shooting numerical calculation method, the transformation constants are evaluated to obtain $m=2.8, l=-1.2$, and the plate Mach number $\mathrm{Ma}=6.510^{-2}$.

The fundamental and the essential inequalities, which we must bear in mind when analyzing the results, are

$$
\begin{gathered}
m_{i} \gg m_{e} \quad \text { makes } \varepsilon^{2}=\frac{m_{e}}{m_{i}}=1.3710^{-5}, \quad \text { (I-a) } \\
\tau_{e e}: \tau_{e i}: \tau_{i i}: \tau_{i e}=1: 7.07 \times 10^{-1}: 2.69 \times 10^{2}: 5.15 \times 10^{4} \\
\Longrightarrow \tau_{e e} \sim \tau_{e i} \ll \tau_{i i} \ll \tau_{i e} .
\end{gathered}
$$

These inequalities will control the major behavior of both electrons and positive ions in the rest of the discussion. Figure 1(a) clarifies that in the course of time the perturbed velocity distribution functions $F_{1}$ and $F_{2}$ approache to the equilibrium velocity distribution function $F_{0}$ at $y=0.003$, for example. The ions are still departing from equilibrium very slowly; see Figure 1(b).

A comparison between Figures 1(a) and 1(b) shows that the collisions of ions with electrons have a very little effect on the form of the ions nonequilibrium distribution function. On the other hand, collisions of electrons with ions have an important effect on the form of the electrons nonequilibrium distribution function, which is in a good agreement with the well-known study of Braginskii [33]. This is because the transfer of momentum from ions to electrons occurs in about the same time $\sim \tau_{e i}$ as the transfer of the energy; hence, ion-electron momentum transfer is small compared with ionion momentum transfer. The transfer of momentum from electrons to ions occurs in a time of the same order $\sim \tau_{e e}$ as electron-electron transfer time, so that collisions of electrons 

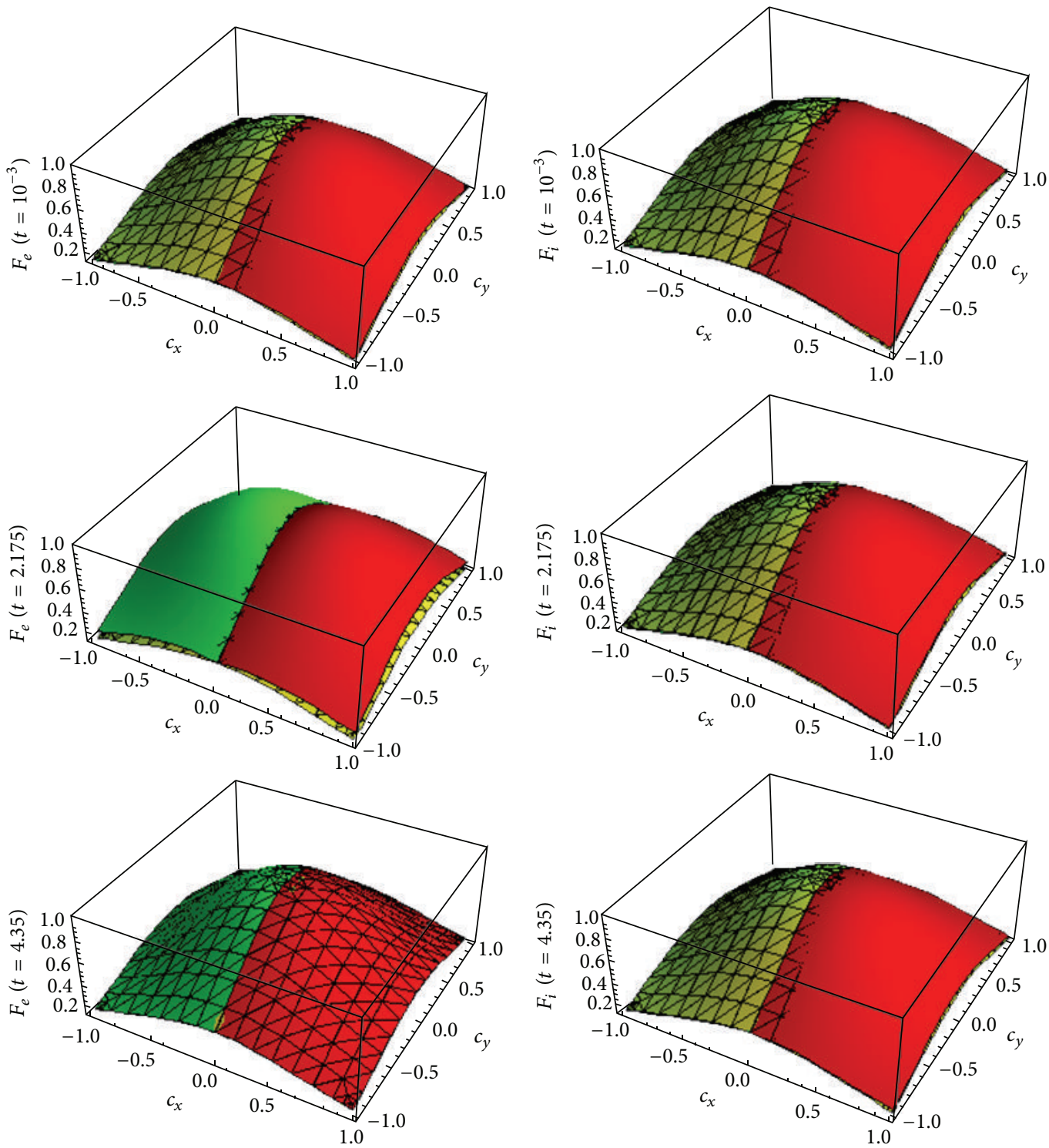

(a)

(b)
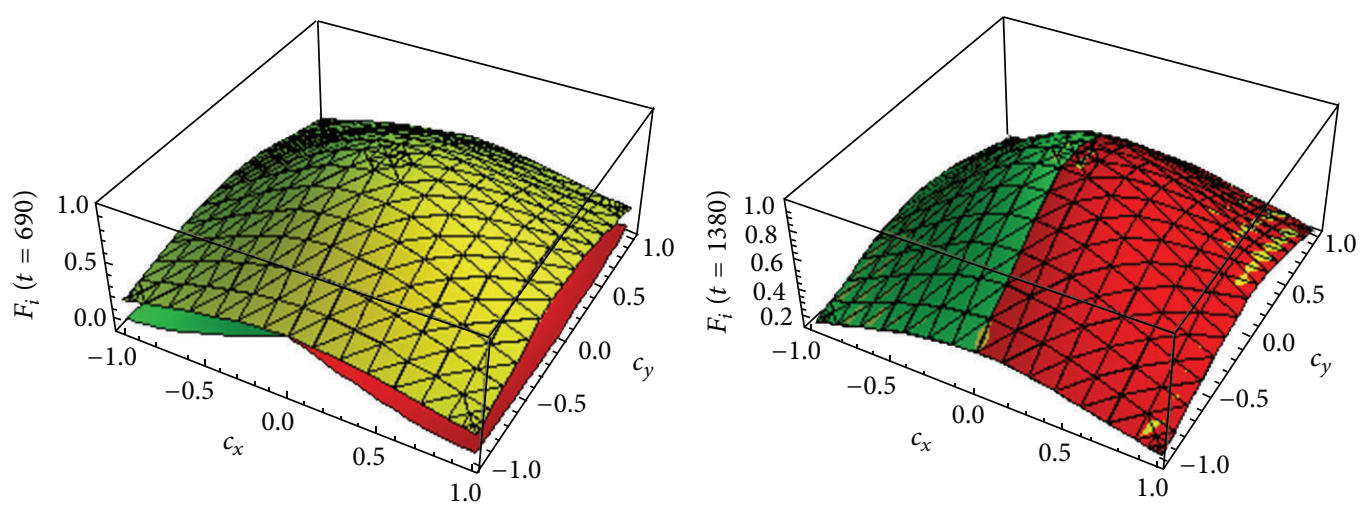

(c)

FIGURE 1: (a) The comparison between the combined perturbed dimensionless velocity distribution functions for electrons $F_{e}\left(F_{1 e}\right.$ (green), $F_{2 e}$ (red)) and electrons equilibrium velocity distribution function $F_{o e}$ (grid) at $t=10^{-3}, 2.175$, and 4.35 for a fixed $y$ value (0.003) with the Mach number of the plate $\mathrm{Ma}=0.065$. (b) The comparison between the combined perturbed dimensionless velocity distribution functions for ions $F_{i}\left(F_{1 i}\right.$ (green), $\left.F_{2 i}(\mathrm{red})\right)$ and ions equilibrium velocity distribution function $F_{o i}$ (grid) at $t=10^{-3}, 2.175$, and 4.35 for a fixed $y$ value ( 0.003 ) with the Mach number of the plate $\mathrm{Ma}=0.065$. (c) The comparison between the combined perturbed dimensionless velocity distribution functions for ions $F_{i}\left(F_{1 i}\right.$ (gray), $F_{2 i}$ (black)) and ions equilibrium dimensionless velocity distribution function $F_{o i}$ (grid) at $t=690,1380$ for a fixed $y$ value (0.003) with the Mach number of the plate Ma $=0.065$. 


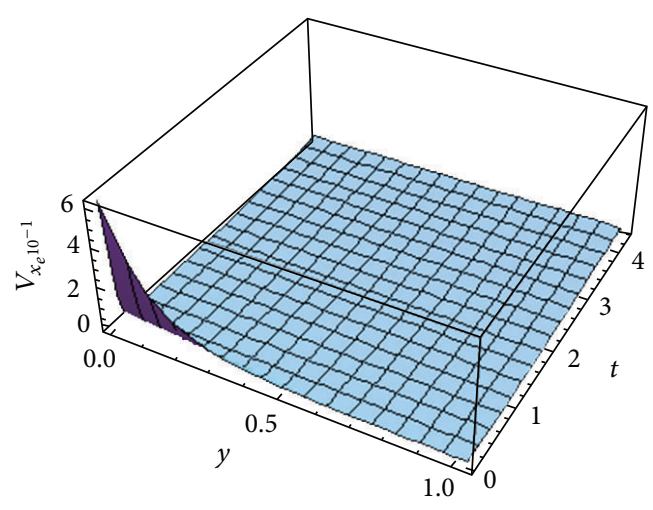

(a)

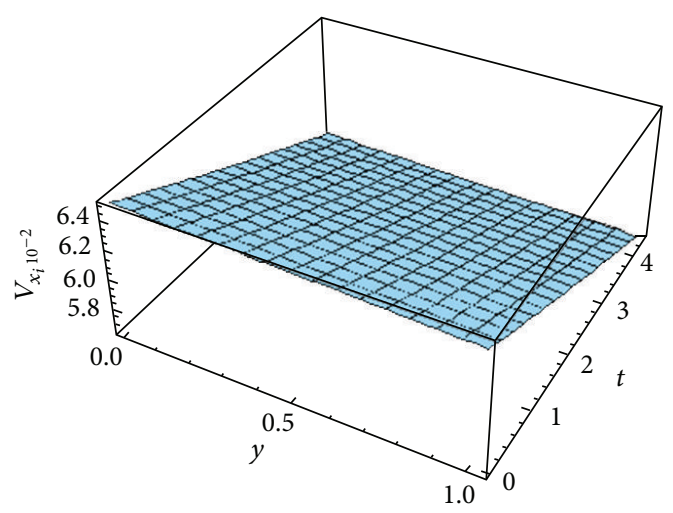

(b)

FIGURE 2: The dimensionless velocity versus space $y$ and time $t$ : (a) $V_{x_{e}}$; (b) $V_{x i}$.

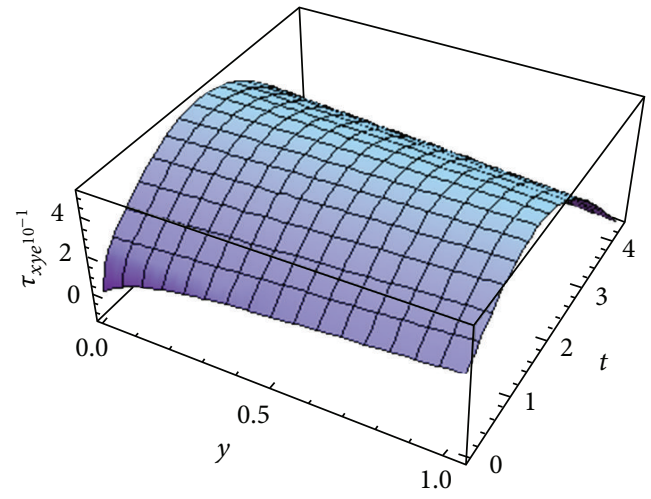

(a)

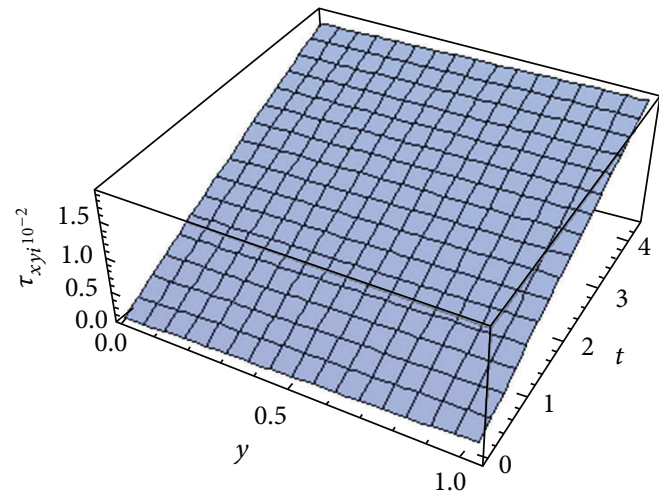

(b)

FIGURE 3: The dimensionless shear stress versus space $y$ and time $t:$ (a) $\tau_{x y e}$; (b) $\tau_{x y i}$.

with the ions affect the electrons nonequilibrium distribution function.

Figures 1(a) and 1(b) indicate that the lighter species (electrons) in the mixture of gases reach equilibrium before the heavier one (positive ions) that is in a qualitative agreement with the investigation made by Galkin [45]. Figure 1(a) makes it clear that the time needed for the electrons to reach equilibrium is $\left(t_{e q} \sim 4.35\right)$ which will be taken into consideration, henceforth.

In a relative long period, for example, $t=690$, the departure of positive ions from equilibrium becomes obvious; the distinction between ions equilibrium distribution function and their nonequilibrium distribution function becomes substantial. After a relatively long time the heavy species (positive ions) reach equilibrium at $t \cong 1380$; see Figure 1 (c).

All Figures 1(a) and 1(b) to 8(a) and 8(b) shed light upon the boundary and initial conditions for both electrons and ions hold. As revealed in Figures 2(a) and 2(b) at the vicinity of the suddenly oscillating plate the mean velocities for both electrons and positive ions have a value $=\mathrm{Ma}$ of the oscillating plate which satisfies the conditions of the problem. The $V_{x e}$ gets a severe decrement with time while $V_{x i}$ shows a gradually linear decrement; this is due to two reasons, firstly, because thermal ion speeds are less than the thermal electron speeds, by the amount $\sqrt{m_{e} / m_{i}}$ as $T_{e} \cong T_{i}$, and so ions take longer time to encounter each other while electrons do not. Secondly, this behavior reflects the fact that the electrons are not very effective in deflecting the much heavier ions, while ions have a very successful effect on electrons motion, referring to inequalities (I-a) and (I-b). Therefore, the change of all kinetic and thermodynamic variables belongs to the positive ions that has unnoticed nonlinear gradually slow changes; see Figures $1(\mathrm{~b})$ to $8(\mathrm{~b})$. This is in a qualitative agreement with the previous paper [15].

The shear stress $\tau_{x y e}$ is beginning from zero value. It is increasing nonlinearly towards its maximum value at $t \sim$ $0.5 t_{\text {eqe }}$. After that, it decreases with the same behavior until it vanishes; see Figure 3(a). This is according to the behavior of the electrons velocity itself. Since the deviation from equilibrium is small, the electron gas is rarefied and the flow is slow. Thus, the gas is Newtonian [46]. It follows that the viscosity $\mu_{\alpha}=-\tau_{x y \alpha} /\left(\partial V_{x \alpha} / \partial y\right)$ represents the resistance to the motion. It gradually increases nonlinearly as the gas particles move away from the plate for ions $\mu_{i}$. The $\mu_{e}$ approaches zero for electrons except at a small time interval around $t \sim 0.5 t_{\text {eqe }}$; this is due to the corresponding maximum value of $\tau_{x y e}$ in the same time interval. 


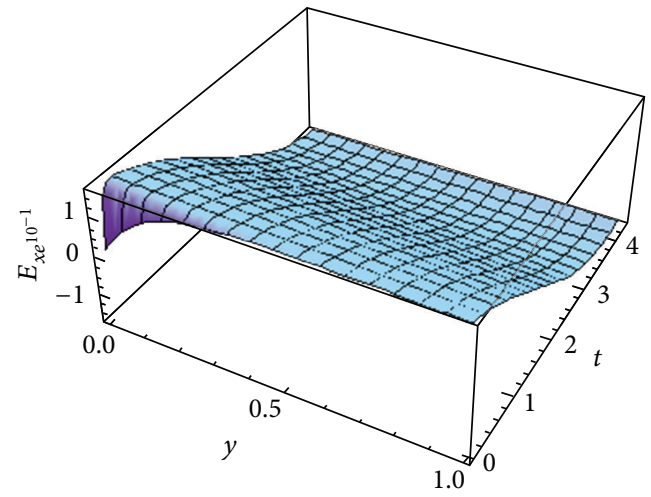

(a)

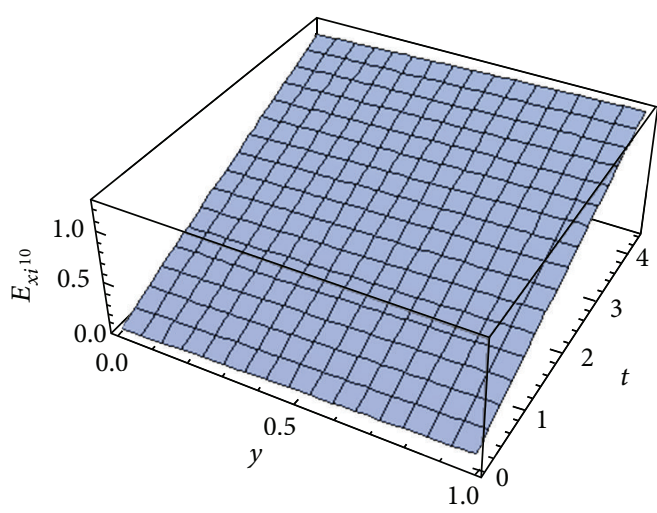

(b)

Figure 4: The dimensionless induced electric field versus space $y$ and time $t$ : (a) $E_{x e}$; (b) $E_{x i}$.

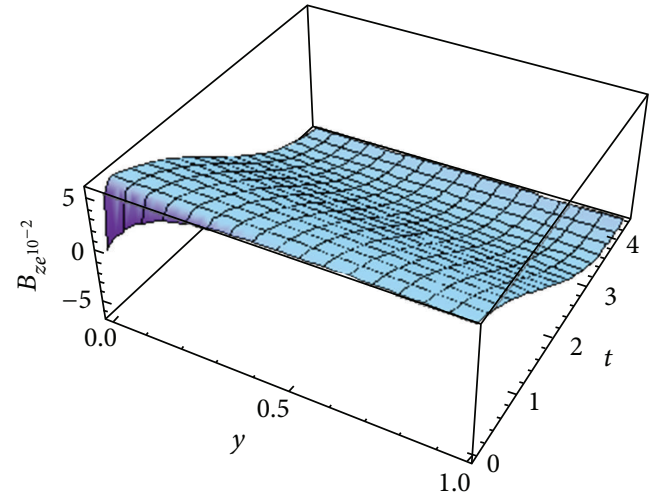

(a)

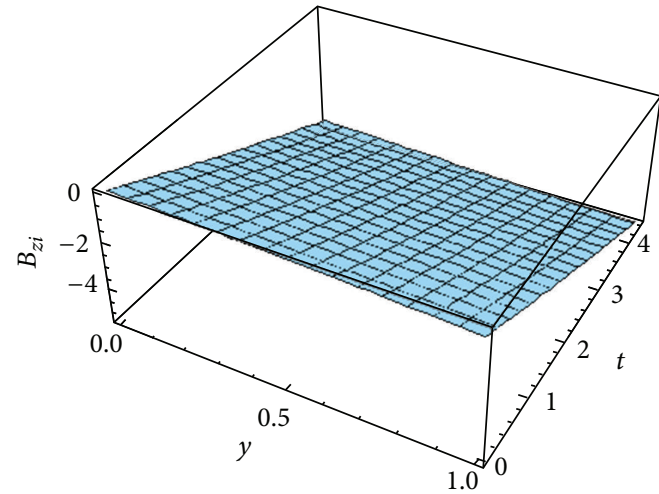

(b)

FIGURE 5: The dimensionless induced electric field versus space $y$ and time $t$ : (a) $B_{z e}$; (b) $B_{z i}$.

The electrons induced electric field has a sudden increase in the beginning until it reaches its maximum value $\left(E_{x e \operatorname{Max}} \sim\right.$ 0.1 ) due to the sudden oscillation of the plate itself. It decreases nonlinearly until it vanishes at $t \sim 0.5 t_{\text {eqe }}$. After that, it changes its direction until the same maximum value $E_{x e \text { Max }} \sim-0.1$ in the opposite direction. It is pushing electrons towards equilibrium. This is because of the famous le Chatelier principle that states, "If the system going away from equilibrium, its particles take the behavior that decreases the departure from equilibrium" that is pushing the system towards equilibrium again; see Figure 4(a). The same behavior holds for electrons induced magnetic field; see Figure 5(a).

Upon passing through a plasma, a charged particle (electron) losses (or gains) part of its energy because of the interaction with the surroundings (positive ions) due to plasma polarization and collisions. The energy loss (or gain) of an electron is determined by the work of the forces acting on the electrons in the plasma by the electromagnetic field generated by the moving particles themselves [47], since the suddenly oscillating plate causes work to be done on the gas, changing the internal energy of the gas $U$. As shown in Figures 6-8, the change in the internal energy due to the variation of entropy and polarization varies smoothly with time by the energy lost to and gained from the ions and plate, respectively. The change in internal energy varies chaotically because of the intensive variables, corresponding to paramagnetic plasma; at the end each tends to zero.

\section{Conclusions}

The solution of the unsteady BGK equation in the case of an inhomogeneous rarefied charged gas, bounded by an oscillating plate, is investigated. We use the method of moments of the two-sided distribution function together with Maxwell's equations. This is developed within the restrictions of small deviation from equilibrium, rarified gas mixture, and slow flow. This solution allows for the calculation of the components of the velocity of the flow for both electrons and positive ions. Inserting them into the suggested two-sided distribution functions and analyzing the results, it is found that:

(a) the lighter species (electrons) of the gas mixture reaches equilibrium before the heave one (positive ions), which is in a qualitative agreement with Galkin [37]; 


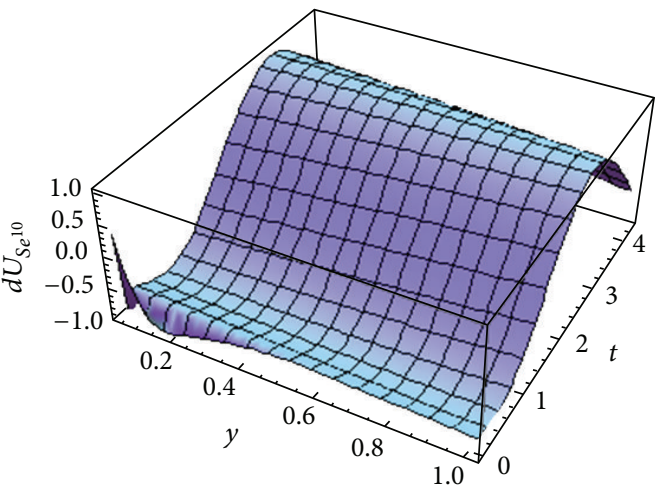

(a)

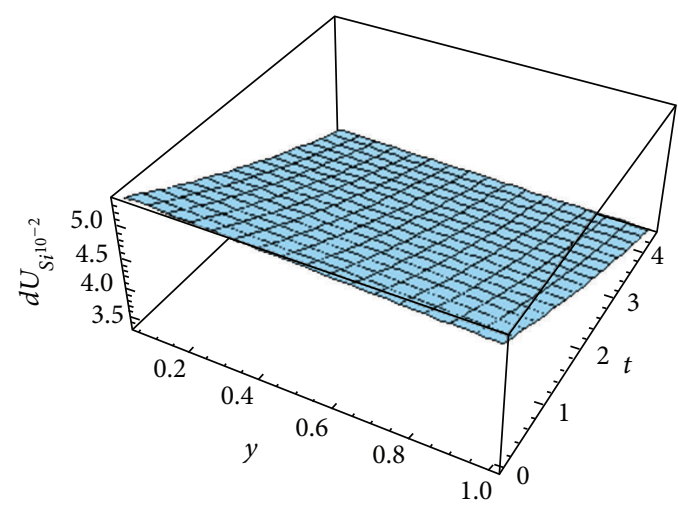

(b)

Figure 6: The dimensionless internal energy change versus space $y$ and time $t$ : (a) $d U_{s e}$; (b) $d U_{s i}$.

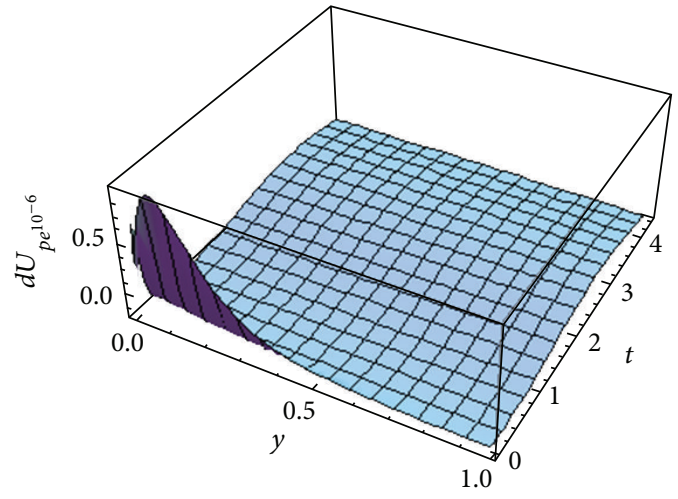

(a)

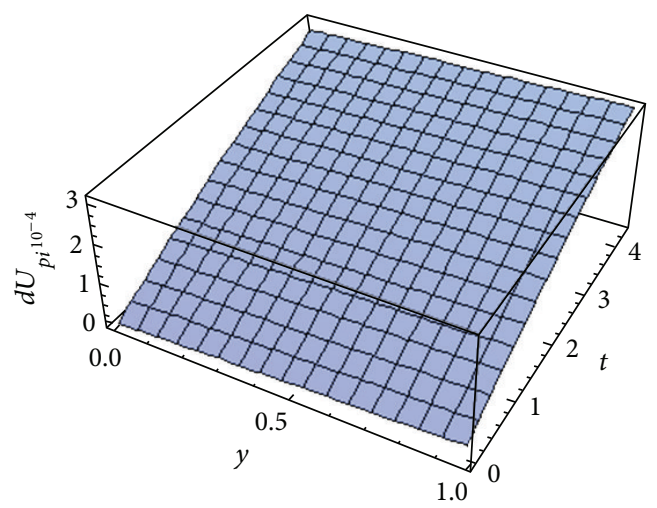

(b)

FIGURE 7: The dimensionless internal energy change versus space $y$ and time $t$ : (a) $d U_{p e}$; (b) $d U_{p i}$.

(b) definitely, the equilibrium times for electrons and for ions are calculated. The relation between those times and the relaxation time for both species of the mixture is deduced. We proved that the collision of ions with electrons has very little effect on the form of the ions nonequilibrium distribution function. On the other hand, we found that the collisions of electrons with ions have an important effect on the form of the electrons nonequilibrium distribution function, which is in a qualitative agreement with the study done by Park et al. [21];

(c) the ratio between the time that electrons $\left(t_{\text {eqe }} \cong 4.35\right)$ and ions $\left(t_{e q i} \cong 1380\right)$ need to reach equilibrium is approximately equal to the same order of the reverse ratio of the ion-ion collision frequency to the mean value of the electron-electron and electron-ion collision frequencies; that is, $t_{e q i} / t_{\text {eqe }} \simeq\left(\left(\left(v_{e e}+v_{e i}\right) / 2\right) / v_{i i}\right)$. This conclusion is different from the situation when the plate is moving with damping velocity and not oscillating; see the previous paper [15].

The predictions, estimated using Gibbs' equation, reveal that the following order of maximum magnitude ratios between the different contributions to the internal energy change based on the total derivatives of the extensive parameters is, for ions

$$
d U_{S i}: d U_{p i}: d U_{\text {pari }}=1: 0.6 \times 10^{-2}: 0.8 \times 10^{-4}
$$

It is concluded that the effect of the changes of the internal energies for positive ions $d U_{p i}$ and $d U_{\text {pari }}$ due to electric and magnetic fields are small in comparison with $d U_{S e}$. This happens with the recognition of the fact that these fields are self-induced by the sudden motion of the oscillating plate.

The same conclusion is applied in the case of electrons such that

$$
d U_{S e}: d U_{p e}: d U_{\text {pare }}=1: 10^{-6}: 10^{-2} .
$$

\section{Nomenclature}

$\vec{B}$ : The induced magnetic field vector

$B$ : The induced magnetic field

$\vec{E}$ : The induced electric vector

$E$ : The induced electric field

$F$ : The velocity distribution function 


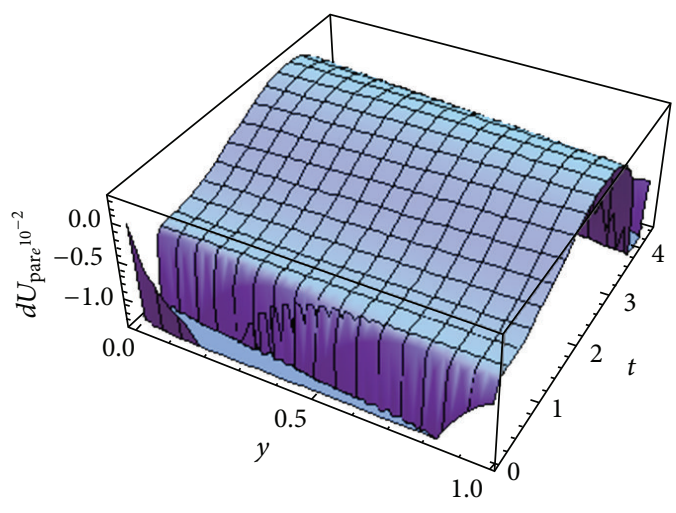

(a)

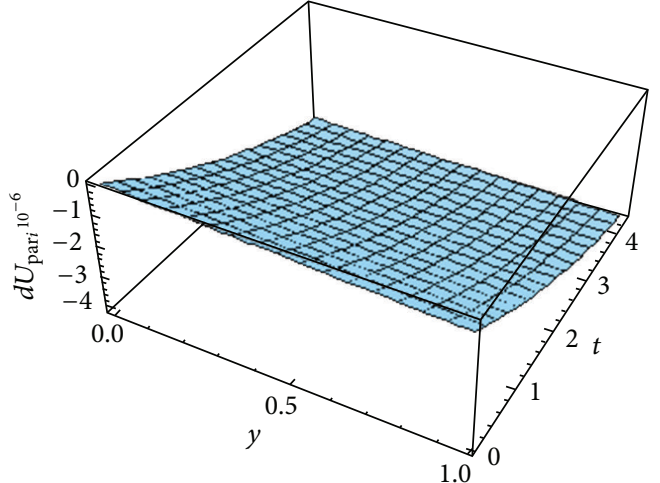

(b)

FIGURE 8: The dimensionless internal energy change versus space $y$ and time $t$ : (a) $d U_{\text {pare }}$; (b) $d U_{\text {pari }}$.

$F_{0}: \quad$ The local Maxwellian distribution function

$F_{1}$ : Distribution function for going downward particles $c_{y}<0$

$F_{2}: \quad$ Distribution function for going upward particles $c_{y}>0$

$J: \quad$ The current density

$K_{B}: \quad$ Boltzmann's constant $\left(\mathrm{Erg} / \mathrm{K}^{0}\right) 1.3810^{-16}$

Ma: The plate Mach number

$M: \quad$ Specific magnetization

$P: \quad$ Polarization

$R: \quad$ The gas constant

$S: \quad$ Entropy per unit mass

T: $\quad$ The temperature

$U: \quad$ The internal energy of the gas

$U_{0}: \quad$ Plate initial velocity

$V_{x}$ : The mean velocity

$V_{x 1}:$ The mean velocity related to $F_{1}$

$V_{x 2}:$ The mean velocity related to $F_{2}$

$V: \quad$ Gas volume

$V_{T e}$ : Thermal velocity of electrons

$V_{T i}:$ Thermal velocity of ions

$c_{0}$ : The speed of light

$c$ : The velocity of the particles

$d$ : $\quad$ Particle diameter

$e: \quad$ The electron charge

$\vec{f}: \quad$ Lorantz's force vector

$m_{e}: \quad$ Electron mass

$m_{i}$ : Ion mass

$n: \quad$ The mean density

$n_{e}: \quad$ Electrons concentration

$n_{i}: \quad$ Ions concentration

p: $\quad$ Pressure

$\vec{r}: \quad$ The position vector of the particle

$t$ : Time variable

$\vec{u}: \quad$ The mean velocity of the particle

$d U_{S}$ : The internal energy change due to the variation of entropy

$d U_{p}$ : The internal energy change due to the variation of polarization

$d U_{\mathrm{par}}$ : The internal energy change due to the variation of magnetization $d U_{\text {dia }}:$ The internal energy change due to the variation of the induced magnetic field

$y$ : $\quad$ Displacement variable

$Z$ : Ionization.

Superscripts

1: Dimensionless variable.

Subscripts

$e$ : Related to electrons

$i$ : Related to ions

$q$ : Equilibrium

$\alpha:=e$ for electrons or $=i$ for ions.

\section{Greek Letters}

$\tau:$ The relaxation time

$\tau_{x y}:$ The shear stress

$\mu$ : Viscosity coefficient

$\lambda$ : The mean free path

$\alpha_{0}$ : Dimensionless parameter

$B$ : Frequency

$v$ : Collision frequency

$\varepsilon$ : Mass ratio

$\lambda:$ Mean free path

$\lambda_{D}$ : Debye radius.

\section{References}

[1] T. Z. Abdel Wahid and S. K. Elagan, "Kinetic treatment for the exact solution of the unsteady Rayleigh flow problem of a rarefied homogeneous charged gas bounded by an oscillating plate," Canadian Journal of Physics, vol. 90, no. 10, pp. 987-998, 2012.

[2] D. D. Millar, "Ion currents, ion-neutral collisions and plasma transport phenomena," Australian Journal of Physics, vol. 29, no. 4, pp. 249-261, 1976.

[3] K. Hyeon-Deuk, "Two-dimensional nonlinear nonequilibrium kinetic theory under steady heat conduction," Physical Review E, vol. 71, no. 4, Article ID 041203, 19 pages, 2005. 
[4] V. V. Aristov, Methods of Direct Solving the Boltzmann Equation and Study of Non-Equilibrium, vol. 60 of Fluid Mechanics and Its Applications, Kluwer Academic, Dordrecht, The Netherlands, 2001.

[5] C. Cercignani, Theory and Application of the Boltzmann Equation, Scottish Academic Press, Edinburgh, UK, 1975.

[6] S. Chapman and T. G. Cowling, The Mathematical Theory of Non-Uniform Gases, Cambridge University Press, Cambridge, UK, 1970

[7] V. P. Shidlovskiy, Introduction to Dynamics of Rarefied Gases, Elsevier, New York, NY, USA, 1967.

[8] G. Lebon, D. Jou, and J. Casas-Vázquez, Understanding NonEquilibrium Thermodynamics: Foundations, Applications, Frontiers, Springer, Berlin, Germany, 2008.

[9] B. C. Eu, Kinetic Theory and Irreversible Thermodynamics, John Wiley \& Sons, New York, NY, USA, 1992.

[10] V. M. Zhdanov and V. I. Roldughin, "Non-equilibrium thermodynamics and kinetic theory of rarefied gases," Physics-Uspekhi, vol. 41, no. 4, pp. 349-378, 1998.

[11] A. M. Abourabia and T. Z. Abdel Wahid, "Solution of the Krook kinetic equation model and non-equilibrium thermodynamics of a rarefied gas affected by a non-linear thermal radiation field," Journal of Non-Equilibrium Thermodynamics, vol. 36, no. 1, pp. 75-98, 2011.

[12] A. M. Abourabia and T. Z. Abdel Wahid, "Kinetic and thermodynamic treatments of a neutral binary gas mixture affected by a nonlinear thermal radiation field," Canadian Journal of Physics, vol. 90, no. 2, pp. 137-149, 2012.

[13] A. M. Abourabia and T. Z. Abdel Wahid, "The unsteady Boltzmann kinetic equation and non-equilibrium thermodynamics of an electron gas for the Rayleigh flow problem," Canadian Journal of Physics, vol. 88, no. 7, pp. 501-511, 2010.

[14] T. Z. Abdel Wahid, "Kinetic and thermodynamic treatment for the exact solution of the unsteady Rayleigh flow problem of a rarefied homogeneous charged gas," Journal of Non-Equilibrium Thermodynamics, vol. 37, no. 2, pp. 119-141, 2012.

[15] A. M. Abourabia and T. Z. Abdel Wahid, "Kinetic and thermodynamic treatment for the Rayleigh flow problem of an inhomogeneous charged gas mixture," Journal of Non-Equilibrium Thermodynamics, vol. 37, no. 1, pp. 1-25, 2012.

[16] G. G. Stokes, On the Effect of the Internal Friction of Fluids on the Motion of Pendulums, vol. 9 of Transactions of the Cambridge Philosophical Society, 1851.

[17] L. Rayleigh, "On the motion of solid bodies through viscous liquid," Philosophical Magazine, vol. 21, no. 126, pp. 697-711, 1911.

[18] X. J. Gu and D. R. Emerson, "Modeling oscillatory flows in the transition regime using a high-order moment method," Microfluidics and Nanofluidics, vol. 10, no. 2, pp. 389-401, 2011.

[19] F. Sharipov and D. Kalempa, "Oscillatory Couette flow at arbitrary oscillation frequency over the whole range of the Knudsen number," Microfluidics and Nanofluidics, vol. 4, no. 5, pp. 363-374, 2008.

[20] F. Sharipov and D. Kalempa, "Gas flow near a plate oscillating longitudinally with an arbitrary frequency," Physics of Fluids, vol. 19, no. 1, Article ID 017110, 2007.

[21] J. H. Park, P. Bahukudumbi, and A. Beskok, "Rarefaction effects on shear driven oscillatory gas flows: a direct simulation Monte Carlo study in the entire Knudsen regime," Physics of Fluids, vol. 16, no. 2, pp. 317-330, 2004.

[22] N. G. Hadjiconstantinou, "Oscillatory shear-driven gas flows in the transition and free-molecular-flow regimes," Physics of Fluids, vol. 17, no. 10, Article ID 100611, 2005.
[23] D. R. Emerson, X. J. Gu, S. K. Stefanov, S. Yuhong, and R. W. Barber, "Nonplanar oscillatory shear flow: from the continuum to the free-molecular regime," Physics of Fluids, vol. 19, no. 10, Article ID 107105, 2007.

[24] T. Doi, "Numerical analysis of oscillatory Couette flow of a rarefied gas on the basis of the linearized Boltzmann equation," Vacuum, vol. 84, no. 5, pp. 734-737, 2009.

[25] P. Taheri, A. S. Rana, M. Torrilhon, and H. Struchtrup, "Macroscopic description of steady and unsteady rarefaction effects in boundary value problems of gas dynamics," Continuum Mechanics and Thermodynamics, vol. 21, no. 6, pp. 423-443, 2009.

[26] W. C. Tang, T. C. H. Nguyen, and R. T. Howe, "Laterally driven polysilicon resonant microstructures," Sensors and Actuators, vol. 20, no. 1-2, pp. 25-32, 1989.

[27] A. Frangi, A. Ghisi, and L. Coronato, "On a deterministic approach for the evaluation of gas damping in inertial MEMS in the free-molecule regime," Sensors and Actuators A, vol. 149, no. 1, pp. 21-28, 2009.

[28] A. H. Khater and A. E. El-Sharif, "Analytical solutions of the Rayleigh flow problem for a rarefied gas of a nonhomogeneous system of charged particles," Physica A, vol. 150, no. 3, pp. 592613, 1988.

[29] A. H. Khater and A. E. El-Sharif, "Analytical solutions of the Rayleigh flow problem for a highly rarefied gas of a homogeneous system of charged particles," Astrophysics and Space Science, vol. 146, no. 1, pp. 157-162, 1988.

[30] E. M. Purcell, Electricity and Magnetism, McGraw-Hill, Singapore, 3rd edition, 1965.

[31] P. L. Bhatnagar, E. P. Gross, and M. Krook, "A model for collision processes in gases. I. Small amplitude processes in charged and neutral one-component systems," Physical Review, vol. 94, no. 3, pp. 511-525, 1954.

[32] J. D. Huba, NRL Plasma Formulary, Navel Research Laboratory, Washington, DC, USA, 2011.

[33] S. I. Braginskii, “Transport processes in a plasma," in Reviews of Plasma Physics, vol. 1, p. 205, Consultants Bureau, New York, NY, USA, 1965.

[34] I. H. Hutchinson, Introduction to Plasma Physics, 2001, http:// silas.psfc.mit.edu/introplasma/index.html.

[35] T. J. M. Boyd and J. J. Sanderson, The Physics of Plasmas, Cambridge University Press, New York, NY, USA, 2003.

[36] H. Grad, "On the kinetic theory of rarefied gases," Communications on Pure and Applied Mathematics, vol. 2, pp. 331-407, 1949.

[37] L. Lees, "Kinetic theory description of rarefied gas flow," Journal of the Society for Industrial and Applied Mathematics, vol. 13, pp. 278-311, 1965.

[38] L. Lees and C. Y. Liu, "Kinetic-theory description of conductive heat transfer from a fine wire," The Physics of Fluids, vol. 5, pp. 1137-1148, 1962.

[39] E. Wasserstrom, C. H. Su, and R. F. Probstein, "Kinetic theory approach to electrostatic probes," Physics of Fluids, vol. 8, no. 1, pp. 56-72, 1965.

[40] A. G. El-Sakka, R. A. Abdellatif, and S. A. Montasser, "Free molecular flow of rarefied gas over an oscillating plate under a periodic external force," Astrophysics and Space Science, vol. 109, no. 2, pp. 259-270, 1985.

[41] J. Gratton, S. M. Mahajan, and F. Minotti, Non Newtonian Gravity Creeping Flow, International Centre for Theoretical Physics, Trieste, Italy, 1988. 
[42] G. Nugroho, A. M. S. Ali, and Z. A. Abdul Karim, "Toward a new simple analytical formulation of Navier-Stokes equations," World Academy of Science, vol. 39, pp. 110-114, 2009.

[43] F. Sharipov, "Reciprocal relations based on the non-stationary Boltzmann equation," Physica A, vol. 391, no. 5, pp. 1972-1983, 2012.

[44] P. Ván, "Thermodynamic stability of dia- and paramagnetic materials," Periodica Polytechnica, vol. 42, no. 2, pp. 97-102, 1998.

[45] V. S. Galkin, "Application of the Chapman-Enskog method to the case of a binary two-temperature gas mixture," Izvestiya Akademii Nauk SSSR. Mekhanika Zhidkosti i Gaza, vol. 2, no. 6, pp. 38-41, 1971.

[46] C. O. Bennett and J. E. Myers, Moment, Heat and Mass Transfer, McGraw-Hill, Singapore, 3rd edition, 1988.

[47] A. G. Sitenko, Electromagnetic Fluctuation in Plasma, Academic Press, New York, NY, USA, 1967. 


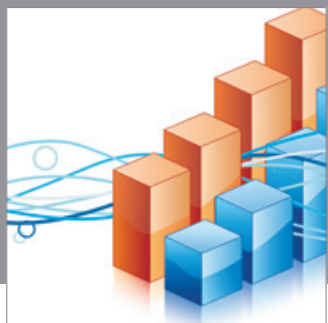

Advances in

Operations Research

mansans

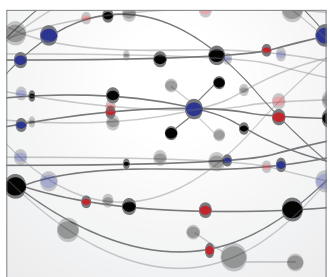

The Scientific World Journal
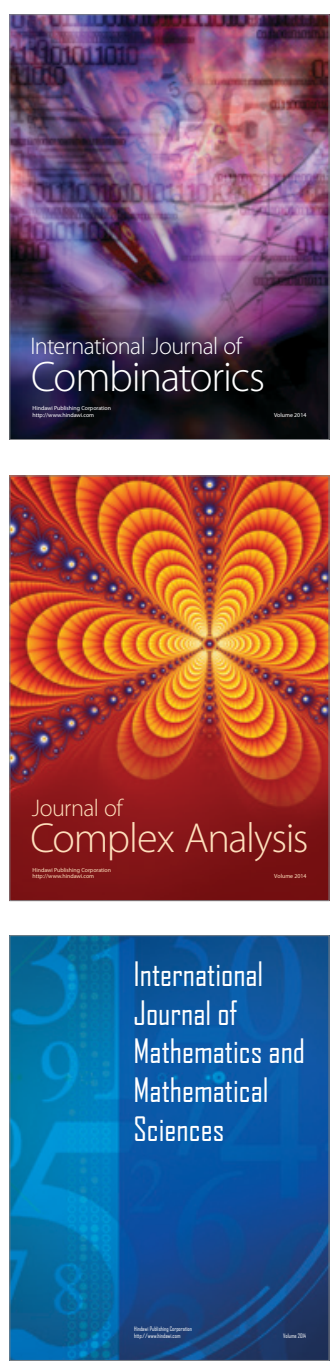
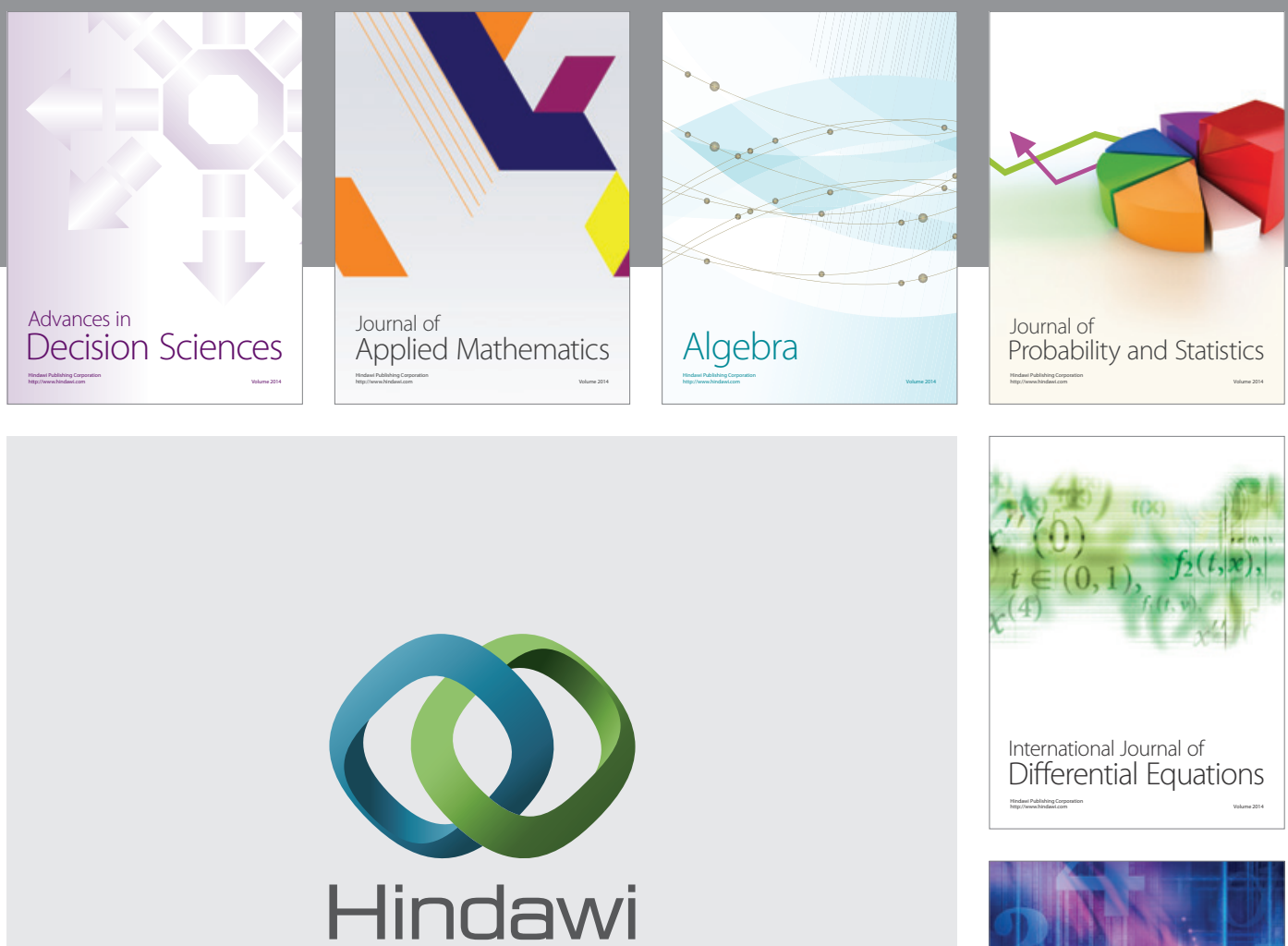

Submit your manuscripts at http://www.hindawi.com
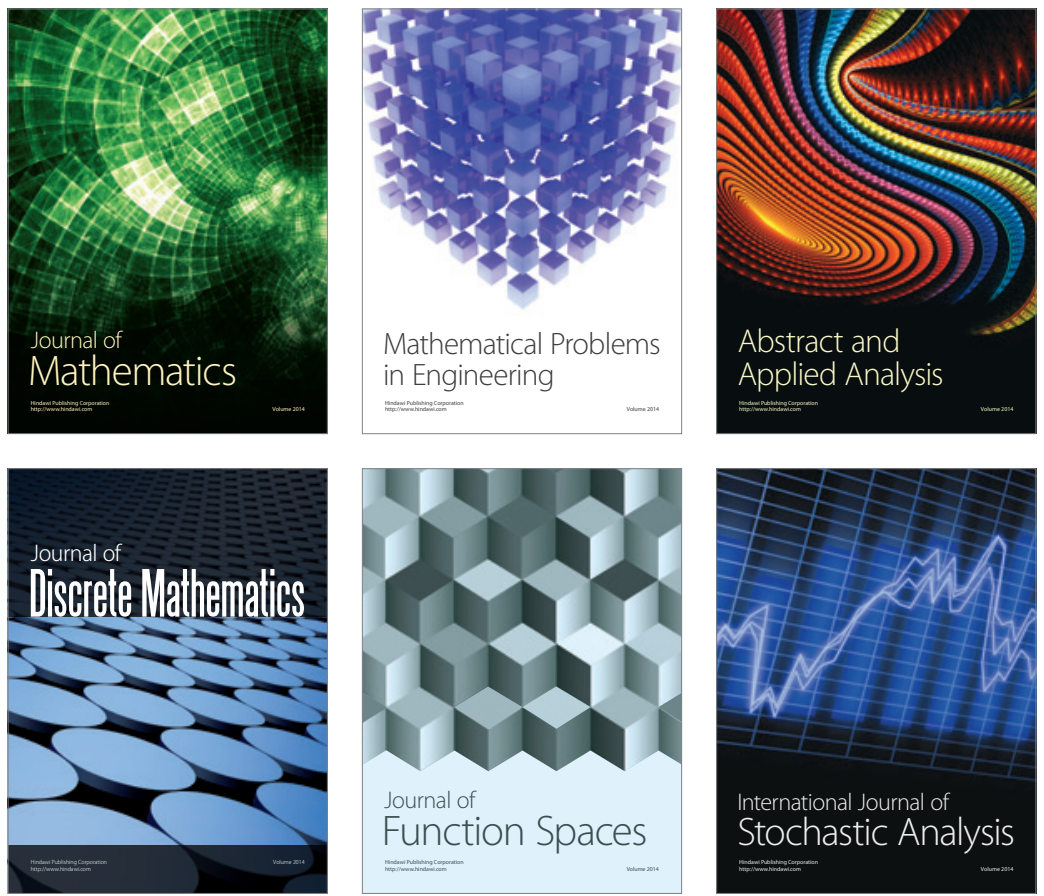

Journal of

Function Spaces

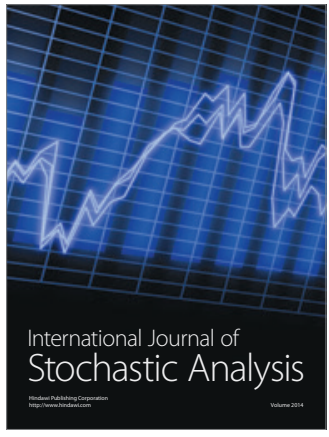

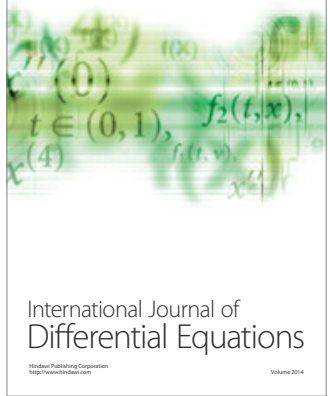
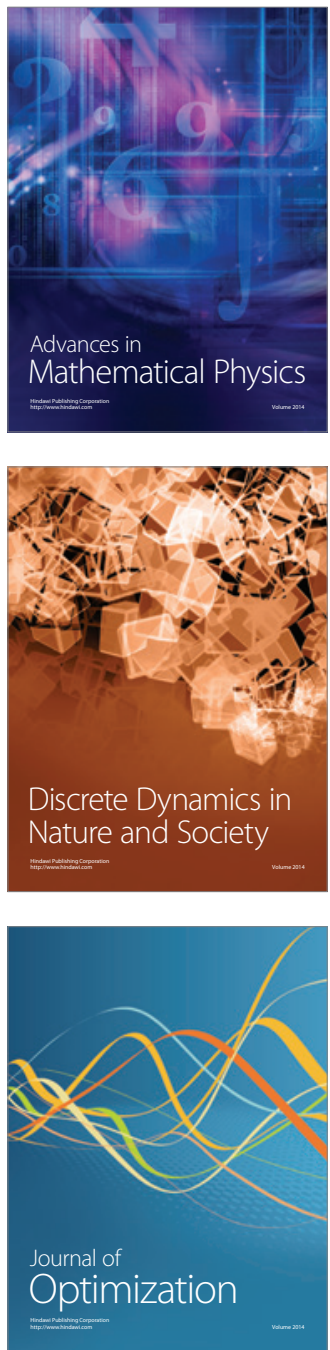